1069

\section{REDUCTION OF NUTRITIONAL DEFICIT MINIMIZE EXTRA UTERINE GROWTH RESTRICTION AMONG EXTREMELY LOW BIRTH WEIGHT INFANTS}

C.-M. Loÿs $\mathbf{s}^{1,2}$, D. Maucort-Boulch ${ }^{3,4}$, B. Guy ${ }^{1}$, J.-C. Picaud ${ }^{1,2}$, G. Putet ${ }^{1,2}$, S. Haÿs ${ }^{1,2}$

${ }^{1}$ Hôpital de la Croix Rousse, Service de Néonatologie et Réanimation Néonatale,

${ }^{2}$ Hospices Civils de Lyon, Université Claude Bernard, ${ }^{3}$ Hospices Civils de Lyon, Service de Biostatistique; Université de Lyon; Université Lyon I, Villeurbanne, ${ }^{4}$ CNRS, UMR 5558, Laboratoire Biostatistique Santé, Pierre-Bénite, Lyon, France

There was a known relationship between cumulative postnatal energy deficit and extra uterine growth restriction experienced by VLBW infants.

Objectives: To determine whether reduction of nutritional deficit had an effect on postnatal growth of ELBW infants born below 28 weeks.

Method: Comparison of postnatal growth between 2 cohorts cared for before (2005) and after (2009) implementation (2006) of a nutrition program that significantly reduced deficit.

Delta z-scores for weight, length and head circumference were calculated each week as the z-scores difference between birth and 36 weeks PMA. Results are reported as median (Q1, Q3). Mann-Whitney test were performed for each criteria.

Results: We compared 33 ELBW infants who experienced nutritional deficit to 32 not exposed to this undernutrition. There was no difference for $G A$ $(p=0.28)$ and BW $(p=0.11)$.

There was a significant reduction of delta z-score for length (- $1.49(-1.93,-0.97)$ versus -0.89 $(-1.76,-0.17), p=0.012)$. Delta $z$-score was neither significantly reduced for weight $(-0.19(-0.79,+0.11)$ versus $-0.03(-0.46,+0.55), p=0.09)$, nor head circumference $(-0.83(-1.26,-0.23)$ versus -0.79 $(-1.47,-0.06), p=0.83)$.

Discussion: This nutrition program allowed reduction of lean mass growth restriction.

Conclusion: Independent effects of energy and protein have to be determined into a multivariate model that also includes non nutritional parameters.

\section{PRECOCIOUS DETECTION OF METABOLIC BONE DISEASE}

N.M. Gianini, S. Dbral, F.C. Barros, L. Bandeira, T.F. Guerreiro, S. Trindade, I. Araguez, G. Monteiro, A.P. Miranda

\section{CETRIN - NICU, Casa de Saúde Santa Lúcia, Rio de Janeiro, Brazil}

Introduction: The improvement of care allowed that LBW infants survive. Among of the routine cares that we must have in the control of these infants it is the detection of the metabolic disease of the prematury (MBD). There are guidelines that use only the serum evaluation of calcium, phosphorus and alkaline phosphatase, excluding the urinary evaluation.

Objective: Comparison of the urinary investigation and the serum about the precocity of the alterations detection that characterize the MBD.

Method: Prospective cohorte study of the newborn admitted in the neonatal intensive care unit in the period of nine (9) months, with low birth weight lower than 1.500 grams. With two weeks of life the urine collection was made for calcium, phosphorus, creatinine (ratio calcium/phosphorus) and calcium, phosphorus and serum alkaline phosphatase.

Results: Sixteen children had been analyzed, with average of weight of birth of $1.263 \mathrm{~g}$ (lower weight: $970 \mathrm{~g}$, greater weight: $1485 \mathrm{~g}$ ). The urinary alterations had been precocious in $62.5 \%$ of the sample of the study. There were not simultaneous urine and blood alterations. With the altered examination the therapeutical treatment was instituted: replacement of calcium and phosphorous via oral administration.

Conclusions: In our cohorte was identified bigger precocity of identification in the compatible laboratorial alteration with MBD when using the urinary investigation of seric eletrolyte. With the findings of the study we decided to use as a routine, only the urinary investigation, which will diminish the number of painful procedures (venous or arterial blood collection) and the withdrawal of sanguine volume. 